\title{
FAUNA ACAROLŌGICA ECTOPARASITA DE SERPENTES NÃO VENENOSAS DA REGIÃO DE CONSTRUÇÃO DE HIDRELÊTRICAS (SUDESTE, CENTRO-OESTE E SUL) DO BRASIL
}

NELIDA M. LIZASO

\begin{abstract}
The ectoparasitic mite fauna of non-poisonous Brazilian snakes, collected in the location of future hydroelectric power plants, is presented. A total of 349 samples of snakes was examined as soon as arrived at the laboratory, to avoid contamination with other parasites, and the numerical host-parasites relationships were analized. Each host presented, in general, a very small number of parasites: hyperparasitism was found once on Waglerophis merremii (Wagler), parasited by Ixobioides and Neotrombicula.
\end{abstract}

\section{INTRODUÇÃO}

A construção de barragens cria um profundo impacto ecológico, que pode ser atenuado em parte pelo planejamento prévio de remoção da fauna atingida pela inundação local. Nos últimos anos, nas barragens construídas no Brasil, este planejamento teve como principal objetivo a devolução dos animais a um ambiente semelhante ao original. Antes do fechamento das comportas procura-se deslocar o maior número possivel de animais; após o fechamento, com a elevação gradual das águas, procede-se à captura dos que vão ficando ilhados para transportá-los a locais pré-estabelecidos, ou retê-los e enviá-los a instituições de pesquisas.

Neste trabalho apresento um estudo do material acarológico ectoparasita de serpentes não venenosas coletadas na área da construção das seguintes barragens:

a) Hidrelétrica de Āgua Vermelha, por represamento do Rio Grande, no limite dos Estados de São Paulo e Minas Gerais;

b) Hidrelétrica de Itumbiara, por represamento do Rio Paranaíba, no limite dos Estados de Goiás e Minas Gerais;

c) Hidrelétrica de Foz do Areia, por represamento do Rio Iguaçu, no sul do Estado do Paraná.

Seção de Artrópodos Peçonhentos, Instituto Butantan, C.P. $65-05504$ São Paulo. Bolsista do Conselho Nacional de Desenvolvimento Científico e Técnico $(\mathrm{CNPq})$. 


\section{MATERIAL E MÉTODOS}

O exame ectoparasitológico das serpentes foi feito imediatamente após sua chegada ao laboratório, para prevenir qualquer possível contágio com parasitas de viveiros ou serpentários. As serpentes foram examinadas depois de anestesiadas com éter sulfúrico para exploração da pele, e os parasitas removidos com escarificador um a um e fixados em álcool $70 \%$.

\section{HIDRELÊTRICA DE ÁGUA VERMELHA}

A construção da Hidrelétrica de Āgua Vermelha foi feita por represamento do Rio Grande (São Paulo) (imediações das coordenadas 50 \% e 20 : S). Após o fechamento das comportas formou-se um reservatório de aproximadamente $570 \mathrm{~km} 2$ de extensão. A inundação foi feita à razão de 1 metro de altura por dia até um total de 35 metros.

Os animais ilhados eram recolhidos em barcos e transportados a lugares apropriados: os terrestres para a Mata de Paulo de Faria, no Estado de São Paulo, adquirida para tal finalidade.

Durante o resgate foram coletadas aproximadamente 1.000 serpentes não venenosas. No período de 5.VII a 15.VIII.78 o Instituto Butantan recebeu uma amostra de 263 exemplares, identificados pelo pessoal da Seção de Herpetologia, com 26 gêneros e 32 espécies, das quais 9 espécies apresentavam-se parasitadas por ácaros (Tabela 1).

Relação dos hospedeiros e seus parasitas

A análise da Tabela 2 revela que:

a) a terça parte dos exemplares de Philodryas olfersii (Lichtenstein) estava parasitada. Esta proporção é mais alta do que verifiquei até agora para o material do Brasil em geral, correspondente a um parasitismo de $5 \%$;

b) um exemplar de Waglerophis merremii (Wagler), serpente bastante abundante na região, estava parasitado só por Amblyomma, o que chama a atenção, pois nesta espécie de serpente é frequente o parasitismo múltiplo, especialmente por Ixobioides.

Analisando a fauna acarológica encontrada nesta amostra são dignos de menção os seguintes dados:

a) de modo geral as serpentes apresentavam pequeno indice de parasitismo; várias delas estavam com um ácaro;

b) Constatei parasitismo múltiplo em 11 serpentes parasitadas por dois gêneros diferentes de ácaros. Esta simultaneidade tem a seguinte distribuição: 
TABELA 1

Serpentes examinadas e parasitadas

\begin{tabular}{|c|c|c|}
\hline SERPENTES EXAMINADAS & $\begin{array}{l}\text { No EXEMPLARES } \\
\text { EXAMINADOS }\end{array}$ & $\begin{array}{l}\text { No EXEMPLARES } \\
\text { PARASITADOS }\end{array}$ \\
\hline Boa constrictor & 27 & 1 \\
\hline Chironius bicarinatus & 1 & - \\
\hline Chironius quadricarinatus & 15 & 2 \\
\hline Chironius flavolineatus & 17 & - \\
\hline Clelia occipitolutea & 6 & - \\
\hline Drymarchon corais & 15 & 4 \\
\hline Elapomorphus nasutus & 1 & - \\
\hline Epicrates cenchria & 20 & 1 \\
\hline Erythrolamprus aesculapii & 1 & - \\
\hline Eunectes murinus & 1 & - \\
\hline Helicops modestus & 4 & - \\
\hline Helicops carinicaudus & 4 & - \\
\hline Hydrodinastes bicinctus & 3 & - \\
\hline Hydrodynastes gigas & 13 & - \\
\hline Leimadophis poecilogyrus & 23 & 2 \\
\hline Leptodeira annulata & 9 & 一 \\
\hline Liophis brazili & 2 & 一 \\
\hline Liophis jaegeri & 2 & - \\
\hline Lygophis meridionalis & 4 & - \\
\hline Lygophis flavifrenatus & 3 & - \\
\hline Mastigodryas bifossatus & 16 & 1 \\
\hline Oxyrhopus petola & 1 & - \\
\hline Oxyrhopus trigeminus & 7 & - \\
\hline Philodryas olfersii & 54 & 19 \\
\hline Philodryas patagoniensis & 1 & - \\
\hline Pseudoboa nigra & 2 & - \\
\hline Rhachidelus brazili & 2 & 一 \\
\hline Sibinomorphus mikanii & 1 & - \\
\hline Simophis rhinostoma & 2 & 2 \\
\hline Spilotes pullatus & 1 & - \\
\hline Thamnodynastes pallidus & 6 & - \\
\hline Waglerophis merremii & 1 & 1 \\
\hline
\end{tabular}

$\begin{array}{llll}\text { Ixobioides } & + & \text { Amblyomma } & 7 \text { exemplares } \\ \text { Ixobioides } & + & \text { Trombiculidae } & 2 \text { exemplares } \\ \text { Ixobioides } & + & \text { Ophioptes } & 2 \text { exemplares }\end{array}$

c) Não encontrei nenhum caso de superparasitismo;

d) 21 serpentes foram encontradas parasitadas por uma única espécie de ácaros. 
TABELA 2

Espécies hospedeiras e seus respectivos parasitas

\begin{tabular}{|c|c|c|c|c|c|}
\hline $\begin{array}{l}\text { NUMERO DE } \\
\text { EXEMPLARES }\end{array}$ & HOSPEDEIROS & 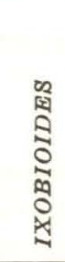 & \begin{tabular}{l} 
o \\
思 \\
0 \\
0 \\
\multirow{2}{*}{} \\
0
\end{tabular} & 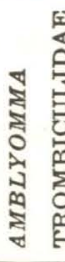 & 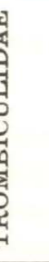 \\
\hline 1 & Boa constrictor & & & $\mathrm{x}$ & \\
\hline 2 & Chironius quadricarinatus & $\mathbf{x}$ & & & \\
\hline 4 & Drymarchon corais & $\mathbf{B}$ & & $\mathrm{x}$ & $\mathbf{x}$ \\
\hline 1 & Epicrates cenchria & $\mathbf{x}$ & & & \\
\hline 2 & Leimadophis poecilogyrus & $\mathrm{x}$ & $\mathbf{x}$ & $\mathrm{x}$ & \\
\hline 1 & Mastigodryas bifossatus & $\mathrm{x}$ & & $\mathrm{x}$ & \\
\hline 19 & Philodryas olfersii & $\mathrm{x}$ & $\mathrm{x}$ & $\mathrm{x}$ & \\
\hline 2 & Simophis rhinostoma & & & & $\mathbf{x}$ \\
\hline 1 & Waglerophis merremii & & & $\mathrm{x}$ & \\
\hline
\end{tabular}

TABELA 3

Serpentes coletadas e parasitadas

\begin{tabular}{ccc}
\hline & No EXEMPLARES & No EXEMPLARES \\
SERPENTES EXAMINADAS & EXAMINADOS & PARASITADOS \\
\hline
\end{tabular}

Boa constrictor

Chironius flavolineatus

1

1

Chironius quadricarinatus

Clelia plumbia

Orymarchon corais

Eunectes murinus

Leimadophis almadensis

Leimadophis reginae

Lygophis meridionalis

Mastigodryaas bifossatus

Oxyrhopus trigeminus

Philodryas olfersii

Philodryas patagoniensis

Simophis rhinostoma

Waglerophis merremii
2

1

2

1

3

1

14

4

5

4

2

2

9

.

1

$\begin{array}{lr}\text { Orymarchon corais } & 1 \\ \text { Eunectes murinus } & 3 \\ \text { Leimadophis almadensis } & 1 \\ \text { Leimadophis reginae } & 14 \\ \text { Lygophis meridionalis } & 4 \\ \text { Mastigodryaas bifossatus } & 5 \\ \text { Oxyrhopus trigeminus } & 4 \\ \text { Philodryas olfersii } & 2 \\ \text { Philodryas patagoniensis } & 2 \\ \text { Simophis rhinostoma } & \mathbf{9} \\ \text { Waglerophis merremii } & \end{array}$


T A B E L A 4

Hospedeiros e parasitas

\begin{tabular}{|c|c|c|c|c|c|}
\hline $\begin{array}{l}\text { NÚMERO DE } \\
\text { EXEMPLARES }\end{array}$ & HOSPEDEIROS & 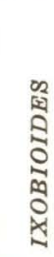 & 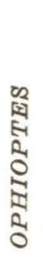 & $\begin{array}{l}\text { J } \\
\text { ป } \\
\text { ป } \\
\text { a } \\
\text { ป }\end{array}$ & 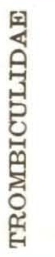 \\
\hline 1 & Chironius flavolineatus & $\mathrm{x}$ & & & 2 \\
\hline 1 & Drymarchon corais & $\mathrm{x}$ & & & \\
\hline 2 & Leimadophis almadensis & $\mathrm{x}$ & & & 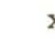 \\
\hline 8 & Leimadophis poecilogyrus & $\mathrm{x}$ & $\mathrm{x}$ & & 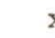 \\
\hline 1 & Leimadophis reginae & $\mathrm{x}$ & & & $x$ \\
\hline 5 & Lygophis meridionalis & $\mathrm{x}$ & $\mathrm{x}$ & & 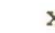 \\
\hline 3 & Oxyrhopus trigeminus & $\mathrm{x}$ & $\mathrm{x}$ & & $x$ \\
\hline 3 & Philodryas olfersii & $\mathrm{x}$ & $\mathrm{x}$ & & $x$ \\
\hline 1 & Philodryas putagoniensis & $\mathrm{x}$ & & & y \\
\hline 2 & Simophis rhonostoma & $\mathrm{x}$ & & & s \\
\hline 8 & Waglerophis merremii & $\mathrm{x}$ & $\mathrm{x}$ & $\mathrm{x}$ & 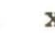 \\
\hline
\end{tabular}

Ixobioides

Trombiculidae

Ixobioides + Trombiculidae

Ixobioides + Ophioptes

Ixobioides + Ophioptes + Trombiculidae

Ixobioides + Trombiculidae + Amblyomma
6 exemplares

4 exemplares

11 exemplares

2 exemplares

11 exemplares

1 exemplar

Relação dos hospedeiros e seus parasitas

Analisando a Tabela 4, tornam-se dignos de menção os seguintes dados:

a) de modo geral as serpentes se apresentavam bastante parasitadas;

b) com um parasita foram encontradas duas serpentes: Drymarchon corais (Binoe) e Philodryas olfersii (Lichtenstein);

c) superparasitismo foi observado em Waglerophis merremii (Wagler), parasitada por Ixobioides e Neotrombicula;

d) o parasitismo múltiplo foi frequente: 13 serpentes se apresentavam parasitadas por dois gêneros de ácaros, 12 serpentes por três gêneros; as restantes estavam parasitadas por um gênero de ácaros;

\section{HIDRELETRICA DE ITUMBIARA}

Por represamento do Rio Paranaiba (imediações das coordenadas 49 : W e 18 : S) formou-se um reservatório de aproximadamente $680 \mathrm{~km} 2$.

As serpentes chegaram ao Instituto Butantan em duas remessas: 23.X e 16.XI.79. 
Dos 63 exemplares examinados, 35 estavam parasitados (Tabela 3).

e) em geral aparecem muitas formas imaturas de Ixobioides em quase todas as serpentes parasitadas;

f) Ophioptes sp. estava parasitando 13 serpentes. Este gênero tem sido coletado em percentagens bastante baixas nas coletas gerais de ácaros, realizadas rotineiramente por mim.

\section{HIDRELETRICA DE FOZ DO AREIA}

Localizada no Médio Iguaçu (imediações das coordenadas $51^{\circ} \mathrm{W}$ e $26{ }^{\circ}$ ) ; a área inundada é de aproximadamente $167 \mathrm{~km} 2$.

O material estudado foi recebido no período compreendido entre 25.IV e 12.V.80, num total de 23 serpentes, das quais 12 parasitadas (Tabela 5).

T A B E L A 5

Serpentes coletadas e parasitadas

$\begin{array}{ccc} & \text { No EXEMPLARES } & \text { No EXEMPLARES } \\ \text { SERPENTES EXAMINADAS } & \text { EXAMINADOS } & \text { PARASITADOS }\end{array}$

Chironius bicarinatus

Liophis miliaris

Oyrhopus petola

1

Pseudoboa haazi

Spilotes pullatus

Sibinomorphus mikanii

Thamnodynastes pallidus

1

Tomodon dorsatus

Waglerophis merremii

1

1

1

1

2

Xenodon neuwiedii

Relação dos hospedeiros e seus parasitas

A análise da Tabela 6 revelou que:

TABELA 6

Relação dos hospedeiros e seus parasitas

\begin{tabular}{|c|c|c|c|c|}
\hline $\begin{array}{l}\text { NÚMERO DE } \\
\text { EXEMPLARES }\end{array}$ & HOSPEDEIROS & 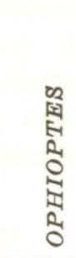 & 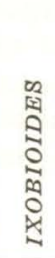 & 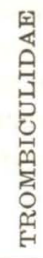 \\
\hline $\begin{array}{l}1 \\
1 \\
2 \\
8\end{array}$ & $\begin{array}{l}\text { Chironius bicarinatus } \\
\text { Oxyrhopus petola } \\
\text { Thamodynastes pallidus } \\
\text { Tomodon dorsatus }\end{array}$ & $\begin{array}{l}\mathrm{x} \\
\mathrm{x} \\
\mathrm{x}\end{array}$ & & \\
\hline
\end{tabular}


a) as serpentes estavam muito pouco parasitadas; um exemplar apresentou 11 ácaros;

b) foram encontradas 3 serpentes com 1 ácaro;

c) cabe destacar que a maioria dos exemplares de Tomodon dorsatus Duméril, Bibron \& Duméril, se encontrou parasitada e sempre por Ixobioides butantanensis. Esta serpente tem mostrado índice baixo de parasitismo nas coletas gerais de ácaros, realizadas por mim rotineiramente.

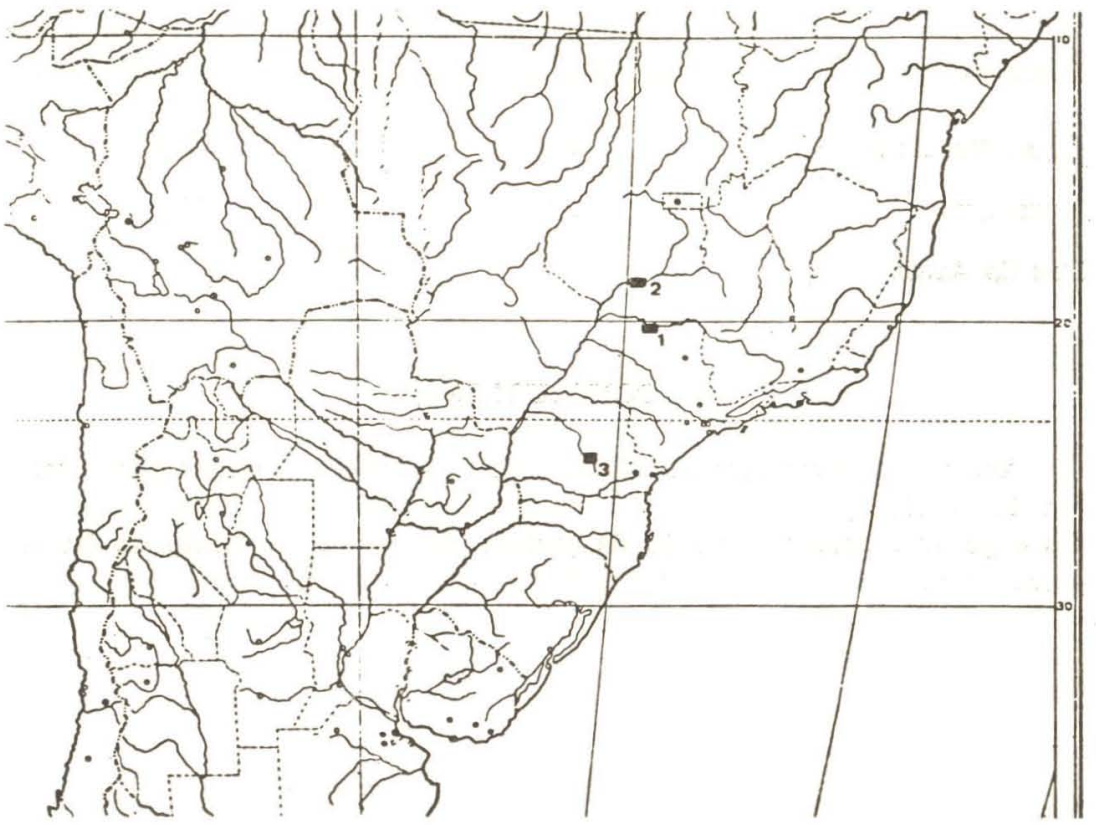

Localização geográfica das Hidrelétricas. 1. Água Vermelha. 2. Itumbiara.

3. Foz do Areia.

Considerações gerais

Comparando o material coletado nestas três hidrelétricas convém destacar:

1. de modo geral encontrei poucos parasitas em cada hospedeiro; constatei um único caso de superparasitismo, em Waglerophis merremii (Wagler), parasitada por Ixobioides e Neotrombicula.

2. O índice de parasitismo em Philodryas olfersii (Liehtenstein) foi de $35 \%$. Nos exames de rotina de coleta para o Brasil, em geral, costuma ser ao redor de $5 \%$. 
3. Também Tomodon dorsatus Duméril, Bibron \& Duméril apresentou índice relativamente elevado: $73 \%$; nas coletas de rotina este índice fica ao redor de $6 \%$; entretanto, o número de parasitas por exemplar foi muito baixo: uma serpente apresentou 11 ácaros, as restantes variaram de 1 a 5 ácaros.

4. Tomodon dorsatus Duméril, Bibron \& Duméril, serpente relativamente comum na região Centro-Sul do Brasil até o norte da Argentina, foi coletada apenas na região da Hidrelétrica de Foz do Areia.

5. Considerando apenas as espécies de serpentes parasitadas, observa-se variação pouco significante em relação à percentagem total das sərpentes coletadas.

Hidrelétrica

Āgua Vermelha

Itumbiara

Foz do Areia
Total de espécies de serpentes parasitadas

$20 \%$

$63 \%$

$70 \%$
Total de espécies de serpentes coletadas

$12 \%$

$55 \%$

$52 \%$

\section{CONCLUSÕES}

Apesar de terem sido analisados dados de três localidades diferentes, em diferentes épocas do ano, os resultados obtidos são bastante completos e poderão servir de ponto de partida para o levantamento da fauna acarológica parasita de serpentes do Brasil. 